\title{
Thymosin $\alpha 1$ therapy subsequent to radical hepatectomy in patients with hepatitis B virus-associated hepatocellular carcinoma: A retrospective controlled study
}

\author{
YONG-RONG LIANG ${ }^{1,2^{*}}, \mathrm{ZHE} \mathrm{GUO}^{3 *}, \mathrm{JING}^{*} \mathrm{HANG} \mathrm{JIANG}^{4}$, BANG-DE XIANG $^{1}$ and LE-QUN LI ${ }^{1}$ \\ ${ }^{1}$ Department of Hepatobiliary Surgery, Affiliated Tumor Hospital of Guangxi Medical University, \\ Nanning, Guangxi 530021; ${ }^{2}$ Department of Hepatobiliary Surgery, Qinzhou First People's Hospital, \\ Qinzhou, Guangxi 535001; ${ }^{3}$ Department of Thyroid and Breast Surgery, The Central Hospital of Wuhan, \\ Wuhan, Hubei 430000; ${ }^{4}$ Department of General Surgery, The Second People's Hospital of Jingmen, \\ Jingmen, Hubei 448000, P.R. China
}

Received April 8, 2015; Accepted August 9, 2016

DOI: $10.3892 / \mathrm{ol} .2016 .5121$

\begin{abstract}
The clinical efficacy of thymosin $\alpha 1$ (T $\alpha 1)$ therapy in patients with hepatocellular carcinoma (HCC) subsequent to radical hepatectomy is unclear. In the present study, the impact of T $\alpha 1$ therapy on outcomes in HCC patients after radical hepatectomy was retrospectively evaluated. Medical records were retrospectively reviewed for 146 patients with hepatitis B virus (HBV)-associated HCC who were treated by radical hepatectomy and subsequently with $\mathrm{T} \alpha 1$ therapy, as well as for 412 control patients with HBV-associated HCC treated by radical hepatectomy. Propensity score matching was used to minimize confounding variables due to baseline differences. Liver function, recurrence-free survival and overall survival rates were compared between the two groups. Serum markers of liver function were significantly improved in the T $\alpha 1$ group compared with the control group. The 1-, 2- and 3-year overall survival rates were 87.2, 82.0 and $68.4 \%$ in the T $\alpha 1$ group and 78.2, 64.2 and $49.7 \%$ in the control group $(\mathrm{P}=0.011)$. The 1-, 2- and 3-year recurrence-free survival rates were 79.7, 70.8 and $67.3 \%$ in the T $\alpha 1$ group and 69.9, 61.5 and $51.6 \%$ in the control group $(\mathrm{P}=0.019)$. The results suggested that post-hepatectomy $\mathrm{T} \alpha 1$ therapy improves liver function
\end{abstract}

Correspondence to: Professor Bang-De Xiang or Professor Le-Qun Li, Department of Hepatobiliary Surgery, Affiliated Tumor Hospital of Guangxi Medical University, He Di Road 71, Nanning, Guangxi 530021, P.R. China

E-mail: xiaopushu-213@163.com

E-mail: zheguophd@163.com

*Contributed equally

Key words: hepatocellular carcinoma, propensity score matching, overall survival, recurrence-free survival rate, thymosin $\alpha 1$ and significantly prolong recurrence-free and overall survival in patients with $\mathrm{HBV}$-associated HCC.

\section{Introduction}

Hepatocellular carcinoma (HCC) is the fifth most common cancer, and it ranks as the third leading cause of cancer-associated mortality worldwide (1). Surgical resection remains the most effective curative treatment for HCC (2), but the high incidence of tumor recurrence makes long-term survival following resection unsatisfactory $(3,4)$. Reducing postoperative recurrence is one of the most significant challenges to improving the prognosis of patients with HCC (5). Several factors $(6,7)$ are hypothesized to affect the risk of recurrence, including whether radical treatment has been performed, what biological characteristics the tumors present, how well the patient's immune system is functioning, and whether the patient has active hepatitis due to hepatitis B virus (HBV) replication. Increasingly, clinicians are exploring ways to boost patient immune function in order to reduce postoperative recurrence (6).

Thymosin $\alpha 1$ (T $\alpha 1)$, a 28-residue peptide hormone with an acetylated $\mathrm{N}$ terminus, is widely used in the clinic to treat patients with chronic HBV infection and to enhance immune response to vaccines in immunocompromised individuals $(8,9)$. Ta1 enhances immunity mainly by inducing the differentiation and maturation of $\mathrm{T}$ cells as well as the production of cytokines $(10)$. Only two studies $(11,12)$ have reported that T $\alpha 1$ may also be effective at reducing risk of postoperative HCC recurrence subsequent to hepatectomy, leading to significantly increased long-term survival rates compared with the absence of T $\alpha 1$ therapy. The reliability of these studies is limited by their relatively small samples (19 and 34 patients) and the fact that the studies did not adjust for potential baseline differences between $T \alpha 1$ and control groups that may have contributed to observed differences in outcomes.

To more rigorously assess the clinical efficacy of postoperative T $\alpha 1$ subsequent to radical hepatectomy in HCC patients, medical records were retrospectively analyzed for recurrence 
and prognosis in a larger cohort of patients treated at the Affiliated Tumor Hospital of Guangxi Medical University (Nanning, China), comparing those who received postoperative T $\alpha 1$ therapy with those who did not. Propensity score matching was used to minimize interference from potential confounders.

\section{Patients and methods}

Patients. The present study was approved by the Ethics Committee of the Affiliated Cancer Hospital of Guangxi Medical University and patients provided consent as part of the standard admission procedure. Medical records were retrospectively analyzed for patients who underwent initial radical hepatectomy for $\mathrm{HCC}$ at the Affiliated Tumor Hospital of Guangxi Medical University between January 2007 and December 2012. To be included, patients had to present with: i) A diagnosis confirmed by postoperative pathological examination; ii) serum hepatitis B e antigen ( $\mathrm{HBeAg}$ ) positivity; iii) a Child-Pugh score of 5-6 for liver function; iv) an Eastern Cooperative Oncology Group score of 0; v) preoperative absence of any cancer embolus in the main portal vein or its first-level branches, the common bile duct or its first-level branches, the main hepatic vein, or the inferior vena cava; vi) absence of intra- and extrahepatic metastases, and total resection of macroscopic tumors without residual cancer cells on the surgical margins; vii) absence of any residual tumor by imaging 1 month subsequent to surgery; and viii) return of $\alpha$-fetoprotein (AFP) levels to the normal range within 2 months of surgery in patients with elevated serum AFP before the operation.

Patients were excluded from the present study if they: i) Had received transarterial chemoembolization (TACE), radiotherapy or other anti-tumor therapies prior to hepatectomy; ii) had undergone emergency surgery; iii) were infected with hepatitis C virus, hepatitis D virus or HIV; iv) had concomitant malignant tumors or severe disorders in other organs; or v) had a history of alcohol or drug abuse.

Patients included in the present study were divided into two groups: Those who received postoperative T $\alpha 1$ therapy and those who did not.

Propensity score matching. Since patients were not assigned randomly to receive postoperative T $\alpha 1$ therapy or not, it was highly likely that the two patient groups would present significant baseline differences that may confound the analysis of outcomes. In order to reduce the bias in patient selection, propensity score matching was performed to balance these baseline differences and thereby simulate random group allocation $(2,13,14)$. Propensity scores were estimated using a logistic regression model based on age, gender, presence of $\mathrm{HBeAg}$, presence of diabetes mellitus, albumin level, total bilirubin level, serum AFP level, alanine aminotransferase (ALT) level, prothrombin time, platelet count, tumor size and number, presence of liver cirrhosis, presence of tumor capsule, microvascular invasion, Edmondson stage, Barcelona Clinic Liver Cancer classification (15), and antiviral therapy administered. Subsequently, 1:1 matching without replacement was performed using a 0.1 caliper width, and the resulting score-matched pairs were used in subsequent analyses, as previously reported (2).
Treatment. Patients in the T $\alpha 1$ group were injected subcutaneously with $1.6 \mathrm{mg}$ T $\alpha 1$ (Chengdu Diao Jiuhong Pharmaceutical Factory, Chengdu, China) twice weekly for 6 months.

Follow-up and outcomes. Control and Ta1 patients returned to the Affiliated Tumor Hospital of Guangxi Medical University for follow-up assessments every 2-3 months. During these visits, serum levels of AFP and the following liver function markers were determined: Albumin level; ALT level; prothrombin time; and total bilirubin level. In addition, imaging tests were performed using B-mode ultrasonography, computed tomography or magnetic resonance imaging. Patients showing the presence of newly developed intra- or extrahepatic tumors consistent with the characteristics of HCC by two imaging modalities were considered to have postoperative recurrence. The outcome measures were postoperative recovery of liver function and recurrence-free and overall survival rates.

Statistical analysis. SPSS 19.0 (IBM, Armonk, NY, USA) was used for statistical analysis. Data with a normal or approximately normal distribution were reported as the mean \pm standard deviation. Data with a non-normal distribution were reported as the median (range). Means, medians and rates between the two patient groups were compared using Student's $t$ test, Mann-Whitney U test or $\chi^{2}$ test, as appropriate. Survival rates were estimated using Kaplan-Meier analysis, and inter-group differences were compared using the log-rank test. The threshold of statistical significance in all cases was defined as $\mathrm{P}<0.05$.

\section{Results}

Patients. A total of 558 patients that met the inclusion criteria were enrolled in the present study, of whom 146 patients received postoperative T $\alpha 1$ therapy and 412 patients did not (Table I). The T $\alpha 1$ and control groups were similar for the majority of baseline characteristics, but there were significant differences in the rates of postoperative antiviral therapy using lamivudine or other drugs, as well as marginal differences in the numbers and longest diameter of tumors. To reduce the risk that these baseline differences may confound the present results, 1:1 propensity score matching was performed to generate 106 pairs that showed no significant differences in any of the baseline parameters (Table I).

Changes in liver function during follow up. The albumin level was significantly higher in the T $\alpha 1$ group compared with the control group at 1,2 and 3 years subsequent to hepatectomy, whereas total bilirubin and ALT levels were significantly lower in the T $\alpha 1$ group at 2 and 3 years (Table II). By 3 years subsequent to surgery, the prothrombin time was significantly shorter in the Ta1 group.

Recurrence-free survival and overall survival analyses. Cumulative recurrence-free survival rates of the Ta1-treated patients in the 106 propensity score-matched pairs were $79.7 \%$ at 1 year, $70.8 \%$ at 2 years and $67.3 \%$ at 3 years subsequent to surgery. The corresponding rates in the 106 control patients were $69.9,61.5$ and $51.6 \%(\mathrm{P}=0.019$; Fig. 1). Cumulative overall survival at the three time points was $87.2,82.0$ and $68.4 \%$ in 


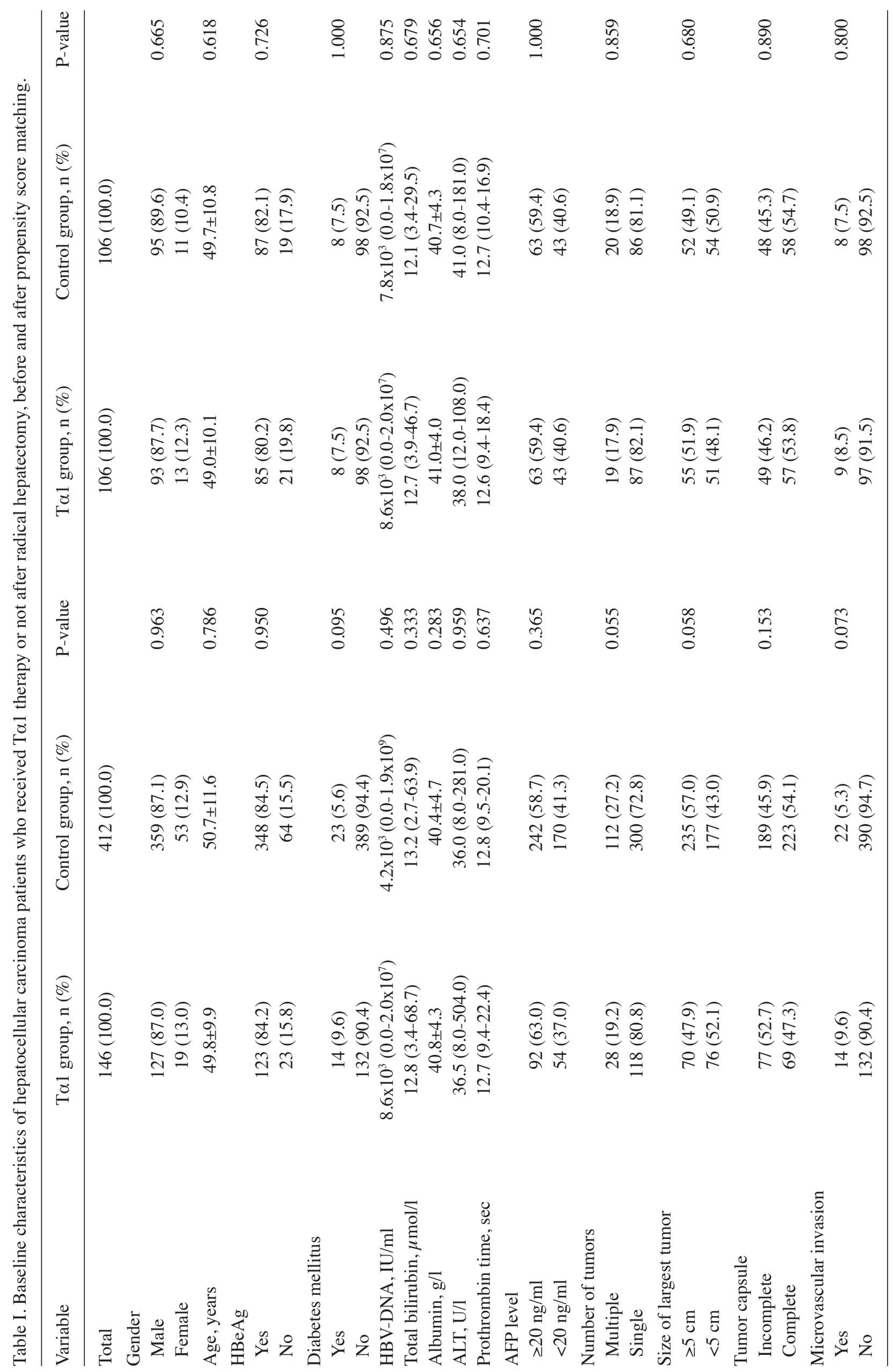




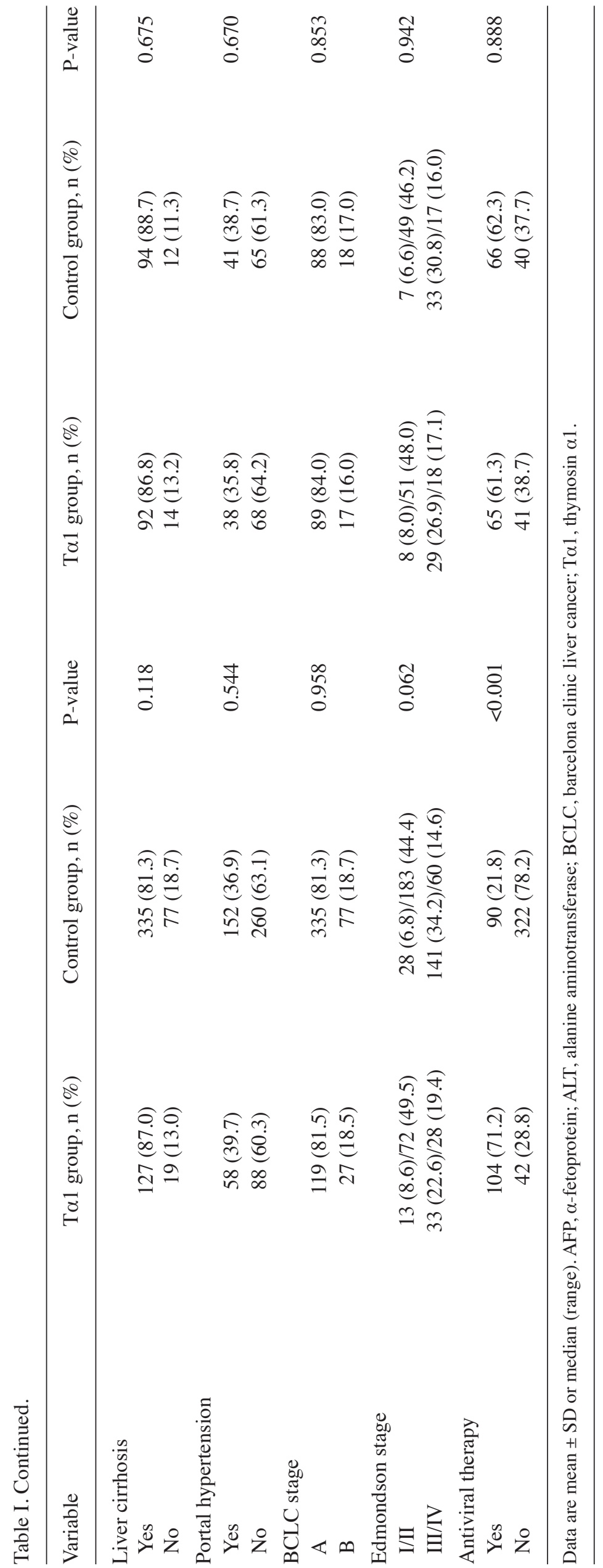


Table II. Post-hepatectomy liver function in hepatocellular carcinoma patients with or without postoperative T $\alpha 1$ therapy.

\begin{tabular}{|c|c|c|c|c|}
\hline Years after surgery & TBIL, $\mu \mathrm{mol} / 1$ & ALB, $g / 1$ & ALT, U/l & PT, sec \\
\hline \multicolumn{5}{|l|}{ Control group } \\
\hline 1 year & $12.4(3.4-506.9)$ & $42.2 \pm 4.9$ & $31.0(8.0-373.0)$ & $13.3(10.3-24.2)$ \\
\hline 2 years & $14.3(5.2-187.6)$ & $39.9 \pm 4.6$ & $33.0(9.0-219.0)$ & $13.3(10.4-17.8)$ \\
\hline 3 years & $13.3(5.8-16.0)$ & $42.3 \pm 4.7$ & $44.5(22.0-65.0)$ & $12.7(11.0-14.1)$ \\
\hline \multicolumn{5}{|l|}{ Ta1 group } \\
\hline 1 year & $10.4(5.0-134.5)$ & $44.0 \pm 4.3^{\mathrm{a}}$ & $31.5(10.0-558.0)$ & $13.0(10.3-19.1)$ \\
\hline 2 years & $10.7(3.8-98.7)^{\mathrm{a}}$ & $45.3 \pm 3.1^{\mathrm{a}}$ & $27.5(6.0-159.0)^{\mathrm{a}}$ & $13.0(10.2-37.9)$ \\
\hline 3 years & $9.9(7.3-12.4)^{\mathrm{a}}$ & $48.6 \pm 2.7^{\mathrm{a}}$ & $31.5(25.0-38.0)^{\mathrm{a}}$ & $11.6(11.2-12.0)^{\mathrm{a}}$ \\
\hline
\end{tabular}

${ }^{\text {a }}<0.05, \mathrm{~T} \alpha 1$ group vs. control group. T $\alpha 1$, thymosin $\alpha 1$; TBIL, total bilirubin; ALB, albumin; ALT, alanine aminotransferase; PT, prothrombin time.

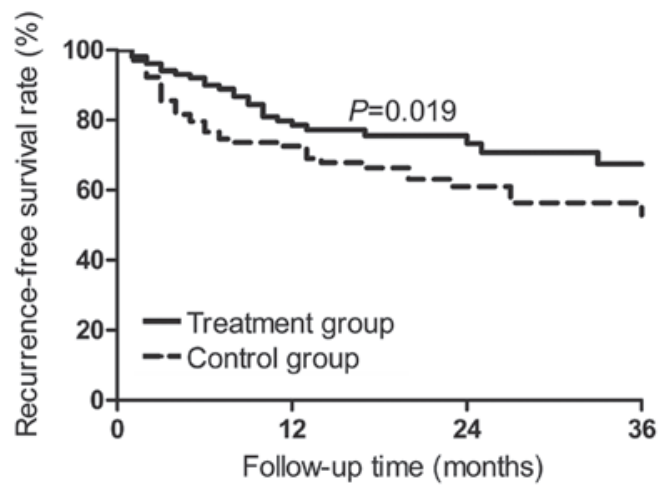

Figure 1. Cumulative recurrence-free survival of propensity score-matched hepatocellular carcinoma patients treated by radical hepatectomy, with or without postoperative thymosin $\alpha 1$ therapy.

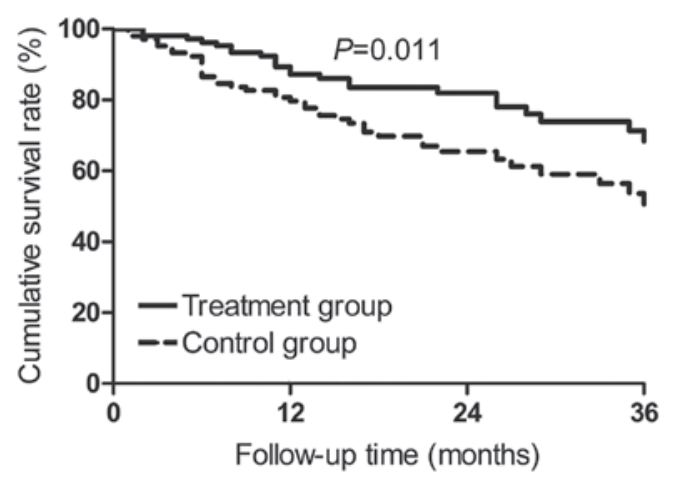

Figure 2. Cumulative overall survival of propensity score-matched hepatocellular carcinoma patients treated by radical hepatectomy, with or without postoperative thymosin $\alpha 1$ therapy.

the propensity score-matched $\mathrm{T} \alpha 1$ patients, compared to 78.2, 64.2 and $49.7 \%$ in the control patients $(\mathrm{P}=0.011$; Fig. 2$)$.

\section{Discussion}

The preferred treatment for early- and intermediate-stage $\mathrm{HCC}$ is radical hepatectomy, but despite impressive advances in early HCC detection and resection techniques, 2-year recurrence remains high, at $45 \%$ (16). Reducing tumor recurrence and/or metastasis subsequent to resection is crucial for the long-term survival of HCC patients. The present study provides evidence that postoperative therapy using the peptide hormone T $\alpha 1$ may significantly increase overall and recurrence-free survival as well as liver function in patients with HBV-associated HCC.

Impaired immunity subsequent to resection is considered to contribute to HCC recurrence both soon afterwards and in the longer term (6). Single-site HCC recurrence appears to be more common within 2 years of surgery, while multi-site recurrence is more common $>2$ years subsequent to surgery (17). The present results, with 3-year recurrence-free survival, suggest that $\mathrm{T} \alpha \mathrm{l}$ can significantly reduce both types of recurrence. T $\alpha 1$ may exert these anti-tumor effects mainly by boosting immune function in several respects (18). T $\alpha 1$ enhances the mitogen-triggered maturation of T $\alpha 1$ lymphocytes in the peripheral blood, increases the secretion of various $\mathrm{T}$ cell lymphokines, such as interferon (IFN)- $\alpha$, IFN- $\gamma$, interleukin (IL)-2 and IL-3, increases the number of lymphokine receptors on the T lymphocyte surface, and activates T4 helper cells to promote lymphocyte activity. T $\alpha 1$ also promotes the chemotaxis of NK precursor cells, thereby increasing cytotoxicity. Previous studies have shown Ta1 to be useful in the treatment of HCC $(11,12)$, and the present study complements that literature by demonstrating significant survival benefits for HCC patients following radical hepatectomy.

In addition to survival rates, postoperative T $\alpha 1$ therapy significantly improved liver function indices, such as total bilirubin, albumin and ALT and prothrombin time, in the present patients. It was hypothesized that in the current patients, T $\alpha 1$ enhanced lymphocyte activity and thereby suppressed viral replication, leading to improved liver function. The Ta1-mediated increase in overall survival was possibly due to the combination of improved liver function and reduction in recurrence.

Strengths of the present study include that it involved larger patient samples than previous studies on this area of research $(11,12)$, and that propensity score matching was used to balance baseline differences between patients who received postoperative Ta1 therapy and those who did not. At the same time, the study has several weaknesses. No data on changes in immune function indices prior and subsequent to 
hepatectomy were included; these indices should be included in future studies. Similarly, data on HBV replication in the present patients was not used, although the observed significant improvement in liver function suggests that T $\alpha 1$ therapy exerted a strong antiviral effect in the present patients.

The present data provide evidence that T $\alpha 1$ therapy may improve overall and recurrence-free survival as well as liver function in HCC patients following radical hepatectomy. The survival benefits of T $\alpha 1$ therapy may be greater than those of other adjuvant therapies, and the therapy may be compatible with a wider range of HCC patients. For example, TACE is effective only in HCC patients at high risk of recurrence (19), while adoptive immunotherapy appears to have little effect on the overall survival rate (6). Interferons may cause adverse reactions such as fever, cold-like symptoms, myelosuppression and hair loss, as well as complications such as hepatic decompensation (20). Lamivudine is indicated only for patients with high serum titers of HBV DNA (21). Future studies should not only verify our findings in patients with $\mathrm{HBV}$-associated $\mathrm{HCC}$, but also examine whether the treatment may benefit other subpopulations of HCC patients.

\section{Acknowledgements}

The authors thank Dr. Armando Chapin Rodríguez for his language editing, which substantially improved the quality of the manuscript. This research was funded by the National Natural Science Foundation of China (grant nos. 81160262 and 81260331), and National Science and Technology Major Project of the Ministry of Science and Technology of China (grant no. 2012ZX10002010001009).

\section{References}

1. Torre LA, Bray F, Siegel RL, Ferlay J, Lortet-Tieulent J and Jemal A: Global cancer statistics, 2012. CA Cancer J Clin 65: $87-108,2015$.

2. Guo Z, Zhong JH, Jiang JH, Zhang J, Xiang BD and Li LQ: Comparison of survival of patients with BCLC stage a hepatocellular carcinoma after hepatic resection or transarterial chemoembolization: A propensity score-based analysis. Ann Surg Oncol 21: 3069-3076, 2014

3. Li T, Fan J, Qin LX, Zhou J, Sun HC, Qiu SJ, Ye QH, Wang L and Tang ZY: Risk factors, prognosis and management of early and late intrahepatic recurrence after resection of primary clear cell carcinoma of the liver. Ann Surg Oncol 18: 1955-1963, 2011

4. Arnaoutakis DJ, Mavros MN, Shen F, Alexandrescu S, Firoozmand A, Popescu I, Weiss M, Wolfgang CL, Choti MA and Pawlik TM: Recurrence patterns and prognostic factors in patients with hepatocellular carcinoma in noncirrhotic liver: A Multi-Institutional analysis. Ann Surg Onco 21: 147-154, 2014.

5. Forner A, Llovet $\mathrm{J}$ and Bruix J: Hepatocellular carcinoma. Lancet 379: 1245-1255, 2012.
6. Zhong JH, Ma L, Wu LC, Zhao W, Yuan WP, Wu FX, Zhang ZM Huang S, You XM and Li LQ: Adoptive immunotherapy for postoperative hepatocellular carcinoma: A systematic review. Int J Clin Pract 66: 21-27, 2012.

7. Lee SC: Prognostic biomarkers for prediction of recurrence of hepatocellular carcinoma: Current status and future prospects. World J Gastroenterol 20: 3112-3124, 2014.

8. You J, Zhuang L, Cheng HY, Yan SM, Yu L, Huang JH, Tang BZ, Huang ML, Ma YL and Chongsuvivatwong V, et al: Efficacy of thymosin alpha-1 and interferon alpha in treatment of chronic viral hepatitis B: A randomized controlled study. World J Gastroenterol 12: 6715-6721, 2006.

9. Kim BH, Lee YJ, Kim W, Yoon JH, Jung EU, Park SJ, Kim YJ and Lee HS: Efficacy of thymosin $\alpha-1$ plus peginterferon $\alpha-2 a$ combination therapy compared with peginterferon $\alpha-2 \mathrm{a}$ monotherapy in $\mathrm{HBeAg}$-positive chronic hepatitis B: A prospective, multicenter, randomized, open-label study. Scand J Gastroenterol 47: 1048-1055, 2012.

10. Matteucci C, Minutolo A, Sinibaldi-Vallebona P, Palamara AT, Rasi G, Mastino A and Garaci E: Transcription profile of human lymphocytes following in vitro treatment with thymosin alpha-1. Ann N Y Acad Sci 1194: 6-19, 2010

11. Cheng SQ, Wu MC, Chen H, Shen F, Yang JH, Zhao YX and Mo ZW: Influence of thymosin $\alpha 1$ on postoperative recurrence of primary liver cancer. Chin J Hepatobil Surg 10: 592-593, 2004 (In Chinese).

12. Palmieri G, Biondi E, Morabito A, Rea A and Bianco A: Could thymostimulin prevent hepatocellular carcinoma occurrence in patients with liver cirrhosis? Oncol Rep 3: 655-656, 1996.

13. Zhong J, Ke Y, Gong W, Xiang BD, Ma L, Ye XP, Peng T, Xie GS and Li LQ: Hepatic resection associated with good survival for selected patients with intermediate and advanced-stage hepatocellular carcinoma. Ann Surg 260: 329-340, 2014.

14. Zhong JH, Xiang BD, Gong WF, Ke Y, Mo QG, Ma L, Liu X and Li LQ: Comparison of long-term survival of patients with BCLC stage B hepatocellular carcinoma after liver resection or transarterial chemoembolization. PLoS One 8: e68193, 2013.

15. Forner A, Reig ME, de Lope CR and Bruix J: Current strategy for staging and treatment: The BCLC update and future prospects. Semin Liver Dis 30: 61-74, 2010.

16. Chun JM, Kwon HJ, Sohn J, Kim SG, Park JY, Bae HI, Yun YK and Hwang YJ: Prognostic factors after early recurrence in patients who underwent curative resection for hepatocellular carcinoma. J Surg Oncol 103: 148-151, 2011.

17. Poon RT: Differentiating early and late recurrences after resection of HCC in cirrhotic patients: Implications on surveillance, prevention, and treatment strategies. Ann Surg Oncol 16: 792-794, 2009.

18. Garaci E, Pica F, Serafino A, Balestrieri E, Matteucci C, Moroni G, Sorrentino R, Zonfrillo M, Pierimarchi P and Sinibaldi-Vallebona P: Thymosin $\alpha 1$ and cancer: Action on immune effector and tumor target cells. Ann N Y Acad Sci 1269: 26-33, 2012.

19. Nitta H, Beppu T, Imai K, Hayashi H, Chikamoto A and Baba H: Adjuvant hepatic arterial infusion chemotherapy after hepatic resection of hepatocellular carcinoma with macroscopic vascular invasion. World J Surg 37: 1034-1042, 2013.

20. Zhong JH, Li H, Li LQ, You XM, Zhang Y, Zhao YN, Liu JY, Xiang BD and Wu GB: Adjuvant therapy options following curative treatment of hepatocellular carcinoma: A systematic review of randomized trials. Eur J Surg Oncol 38: 286-295, 2012.

21. Wu C, Chen Y, Ho HJ, Hsu YC, Kuo KN, Wu MS and Lin JT: Association between nucleoside analogues and risk of hepatitis $\mathrm{B}$ virus-related hepatocellular carcinoma recurrence following liver resection. JAMA 308: 1906-1913, 2012. 